\title{
New anomalous exchange in Regge phenomenology and hard diffraction
}

\author{
$\underline{\text { Nikolai I. Kochelev }}^{\mathrm{a} *}$, Dong-Pil Min ${ }^{\mathrm{b} \dagger}$, Yongseok $\mathrm{Oh}^{\mathrm{c} \ddagger}$, Vicente Vento ${ }^{\mathrm{d} \S}$, Andrey V. Vinnikov ${ }^{\mathrm{q}}$ \\ a'BLP, JINR, 141980 Dubna, Russia \\ ${ }^{\mathrm{b}}$ Department of Physics, Seoul National University, Seoul 151-742, Korea \\ ${ }^{\mathrm{c}}$ Institute of Physics and Applied Physics, Yonsei University, Seoul 120-749, Korea \\ ${ }^{\mathrm{d}}$ Departament de Física Teòrica and Institut de Física Corpuscular, Universitat de València-CSIC \\ E-46100, Burjassot (Valencia), Spain \\ ${ }^{\text {e}}$ Far Eastern State University, Sukhanova 8, GSP, Vladivostok, 690660 Russia

\begin{abstract}
A new mechanism for hard diffraction based on the anomalous $f_{1}$ trajectory exchange, which we identify as the
\end{abstract} \\ odd signature partner of the Pomeron, is suggested. We calculate the contribution of the $f_{1}$ exchange to elastic \\ and dissociative electromagnetic production of vector mesons and show that it gives a dominant contribution to \\ the differential cross sections at large momentum transfers.
}

\section{Introduction}

Understanding the mechanisms of diffraction at large energies is one of the main topics in QCD 11. This interest comes from the possibility that the fundamental problems of QCD in the diffractive processes are related to the perturbative and nonperturbative quark-gluon interactions. One of such problems is the role of the complex structure of $\mathrm{QCD}$ vacuum in parton scattering. A well-known fact is that at large energies the dominant contribution to the parton-parton scattering cross sections comes from the exchange which has the vacuum quantum numbers. At small momentum transfers this exchange can be successfully described by the famous Pomeron which has $P=C=+1$ and even signature. If the virtualities of the quarks and gluons are large enough, then the hard BFKL Pomeron, based on pQCD, would play an important role. When the virtualities of the partons are small, the QCD vacuum structure becomes very important, where

\footnotetext{
*e-mail: kochelev@thsun1.jinr.ru

${ }^{\dagger}$ e-mail: dpmin@mulli.snu.ac.kr

‡e-mail: yoh@phya.yonsei.ac.kr

$\S$ e-mail: Vicente.Vento@uv.es

ๆ e-mail: vinnikov@thsun1.jinr.ru
}

the soft Pomeron contribution is dominant phenomenologically. The relations between the soft Pomeron properties and the QCD vacuum structure are widely under discussion 22. The most interesting idea is relating the unusual properties of the Pomeron trajectory, e.g., its high intercept and small slope, with the existence of the scale anomaly in QCD [2].

There is another class of diffractive process, i.e., the hard diffraction at large $t$ and small virtualities of initial and final particles, which has been much less studied experimentally and theoretically. In this process, colourless exchanges between partons are involved having large momentum transfers. The simplest example of such reactions is elastic hadron-hadron scattering at large $t$. It should be mentioned that the first data from ISR on $p p$ elastic scattering at large $t$ have already shown some unexpected results, e.g., only one diffractive minimum was observed instead of the set of minima anticipated from the multi-Pomeron exchange picture. In order to explain such phenomena, the Odderon exchange hypothesis, which has $P=C=-1$ and intercept $\alpha_{O}(0) \approx 1$, has been suggested [8]. The peculiarity of such exchange is in its negative charge 
parity, which should lead in particular to the difference between the $p p$ and the $p \bar{p}$ scattering cross sections at large energies. However it was also claimed that the modern data on $p p$ and $p \bar{p}$ elastic scattering could be explained without introducing the Odderon exchange at least at $t=0$ [3].

New data on large $t$ hard diffraction are now available from HERA [ [ [6]. These data show unusual energy dependence of the cross sections for vector meson electromagnetic production and thus call for new ideas as they are difficult to explain within the conventional mechanisms based on the soft Pomeron exchange. Recently we suggested a mechanism [7] to understand these data by introducing a new anomalous $f_{1}$ exchange in Regge theory, which corresponds to an effective trajectory with a high intercept $\alpha_{f_{1}}(0) \approx 1$ and small slope $\alpha_{f_{1}}^{\prime} \approx 0$. This exchange has the quantum numbers of the flavor-singlet axial-vector $f_{1}(1285)$ meson, i.e., $P=C=+1$ and odd signature, and can be considered as the natural partner of another anomalous Regge trajectory, i.e., the Pomeron, which has even signature and the same parities $P=C=+1$. Moreover we have shown that the $f_{1}$ exchange allows us to understand the anomalous behavior of the spin-dependent structure function $g_{1}(x)$ at low $x$ and the universal behavior of the differential cross sections for elastic $p p$ and $p \bar{p}$ scattering at large momentum transfers. The quantum numbers of this trajectory $(P=C=+1)$ are different from those of the Odderon $(P=C=-1)$ [8] and can be verified by experimental measurements on several spindependent cross sections [7,9].

\section{Elastic electromagnetic production of vector mesons and $f_{1}$ exchange}

Among the possible effective quark-quark interactions, there are only two kind of vertices, $\gamma_{\mu} \otimes \gamma_{\mu}$ and $\gamma_{\mu} \gamma_{5} \otimes \gamma_{\mu} \gamma_{5}$, which survive at high energies and whose Lorentz structure does not lead to the quark helicity-flip process at large energy $s$ and small momentum transfer $-t \ll s$. The spin-flip property of the quark-quark scattering is very important for the high energy behavior of the corresponding scattering amplitude because any spin-flip amplitude should be suppressed by additional factors $t / s$ due to total angular momentum conservation. Therefore only the above mentioned Lorentz structures can lead to nonvanishing cross sections at large energies in the forward scattering limit.

The vector-like structure $\gamma_{\mu} \otimes \gamma_{\mu}$ has been suggested many years ago as a possible effective interaction induced by the soft Pomeron exchange 11. This structure has enjoyed much success in the description of many hadron-hadron, lepton-hadron and photon-hadron reactions at small $|t|$ region $\left(|t| \leq 1 \mathrm{GeV}^{2}\right)$, as well as their total cross sections, once the phenomenological Pomeron trajectory and form factors are used. However the possible existence of the other important structure $\gamma_{\mu} \gamma_{5} \otimes \gamma_{\mu} \gamma_{5}$ has not been noticed seriously until Ref. [7]. It should be emphasized that the effective interactions for both anomalous exchanges have the structure of the product of two vector or axial-vector currents: $J_{\mu}^{P} \otimes J_{\mu}^{P}$ for the Pomeron and $J_{5 \mu}^{a} \otimes J_{5 \mu}^{a}$ for the $f_{1}$ exchange. The similarity of the Lorentz structure of both exchanges has probably a deep origin. The Pomeron current is expected to be connected with the non-conserved dilaton current whose divergence is given by the scale anomaly, and the $f_{1}$ anomalous exchange is related to the nonconserved axial vector current whose divergence is given by the axial anomaly [7]. The connection between these anomalous exchanges would also be natural in SUSY QCD where only some definite chiral combinations $G^{2} \pm i G \widetilde{G}$ correspond to superfields [12], which leads to an expectation that these trajectories are degenerate in SUSY QCD.

On the hadronic level the anomaly manifests itself in unusual decay rates. The famous example is the axial anomaly in $\pi^{0} \rightarrow \gamma \gamma$ decay due to triangle graph contribution to the transition amplitude. The large mass of the $\eta^{\prime}$ meson is also related to the large mixing of this flavor singlet with gluons due to axial anomaly. Recently it was suggested that abnormal properties of Pomeron trajectory would be explained by the large mixing between glueball and $q \bar{q}$ trajectories 10 . In the axial vector channel the lowest meson state with the appropriate quantum numbers, $I=0$ and $P=C=+1$, is the $f_{1}(1285)$. 
This flavor-singlet axial vector meson can couple to the quarks through mixing with a two gluon state due to triangle anomaly. In accordance with the Landau-Yang theorem the coupling of $1^{++}$ state to two gluon state vanishes for on-shell gluons. Therefore the intermediate gluons should be highly virtual to give a contribution to the $f_{1} q q$ coupling and we thus consider $f_{1} q q$ vertex as point-like.

The diffractive electromagnetic production of vector mesons is one of the appropriate processes for testing different perturbative QCD and QCDinspired nonperturbative models for quark-quark scattering. Recently new experimental data on photo- and electro-production of $\rho, \omega$ and $\phi$ mesons at large energyies $W \approx 100 \mathrm{GeV}$, and large momentum transfers, up to $-t \approx 2 \mathrm{GeV}^{2}$ in elastic production and up to $-t \approx 11 \mathrm{GeV}^{2}$ in low-mass dissociation, have been reported [4,5]. At small $|t|$ and large $W$, the fixed-target photoproduction data have been described successfully within the soft Pomeron exchange models with the universal Pomeron trajectory $\alpha_{P}^{\text {soft }}(t)=$ $1.08+0.25 t$. But the reported new data violate this universality at large $|t|$ region 任. In order to describe such observations in terms of Regge trajectories, an additional Pomeron, called hard Pomeron, with the trajectory $\alpha_{P}^{\text {hard }}=1.4+0.1 t$ has been proposed [13]. However it is not yet certain whether this approach can also describe the energy independence of the elastic $p p$ and $p \bar{p}$ cross sections at large energies and momentum transfers.

In a recent paper [7] we have shown that the anomalous $f_{1}$ exchange can describe both the elastic hadron-hadron and $\rho / \phi$ photoproduction cross sections in a consistent way. In the calculation of the $f_{1}$ exchange contribution to $\rho$ and $\phi$ photoproduction, we have assumed a specific phenomenological form factor in the $f_{1} V \gamma$ vertex, which we would like now to relax. Furthermore the strength of this vertex was fixed from the decay width of $f_{1} \rightarrow \gamma V$, which is a questionable procedure for parameters to be used in high energy scattering where the quark structure of the hadrons is important. Moreover the absence of experimental data on the $f_{1} \rightarrow \gamma \omega$ decay did not allow us to estimate the $f_{1}$ exchange contribution

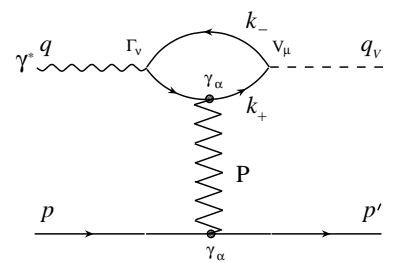

Figure 1. Pomeron exchange contribution to vector meson production.

to $\omega$ meson photoproduction.

In this work we use a method similar to that used to calculate the Pomeron exchange contribution to vector meson production in Ref. [11]. We then generalize the approach to include the $f_{1}$ exchange process in the same production amplitude. One important feature of this approach is that we have less parameters for photoproduction and that it can be generalized straightforwardly to electroproduction. The Pomeron exchange contribution in the electromagnetic production of vector mesons is presented in Fig. 1. The soft Pomeron contribution to transverse virtual photoproduction cross section at large energy reads

$$
\begin{aligned}
\frac{d \sigma_{P}^{T}}{d t}= & \frac{27 m_{V}^{3} \beta_{u}^{2} \beta_{f}^{2} \Gamma_{e^{+} e^{-}}^{V}\left(\frac{s}{s_{0}}\right)^{2 \alpha(t)-2}}{\pi \alpha_{\mathrm{em}}} \\
& \times\left[\frac{F_{1}(t) \sqrt{1-t / 2 m_{V}^{2}}}{\left(Q^{2}+m_{V}^{2}-t\right)}\right]^{2} F\left(t, Q^{2}\right),
\end{aligned}
$$

where the factor $F\left(t, Q^{2}\right)$ accounts for the nonlocality of the Pomeron-quark vertex.

The contribution of the $f_{1}$ exchange to vector meson electromagnetic production (Fig. 2) is obtained in a similar manner as in the calculation of the Pomeron exchange amplitude. The main difference lies on the substitution of $\gamma_{\alpha}$ matrix in the effective Pomeron-quark vertex by $\gamma_{\alpha} \gamma_{5}$ in the effective $f_{1}$-quark vertex. Note that we do not have any new free parameters for this exchange since the coupling constant $g_{f_{1} f f}$ is fixed by

$g_{f_{1} f f}=g_{f_{1} N N}$,

which can be obtained using a constituent quark picture for the nucleon wave function. It should 


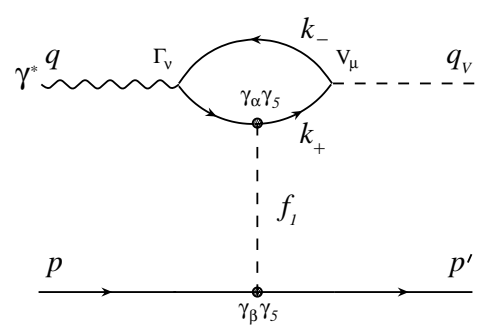

Figure 2. $f_{1}$ exchange contribution to vector meson production.

be mentioned that this relation is different from the additivity of the Pomeron coupling, i.e., $g_{P N N} \approx 3 g_{P q q}$. What Eq. (2) implies is that the axial vector coupling is sensitive only to the spin of the particle. In our calculation we use $g_{f_{1} N N}=2.5$ that is obtained from the analyses of the $f_{1}$ contribution to the proton spin (see Ref. (7]). Then the final form for the $f_{1}$ exchange contribution to transverse virtual photoproduction cross section is (for $\alpha_{f_{1}}(0)=1$ and $\alpha_{f_{1}}^{\prime}=0$ )

$$
\begin{aligned}
\frac{d \sigma_{A}^{T}}{d t}= & \frac{3 m_{V}^{3} g_{f_{1} N N}^{4} \Gamma_{e^{+} e^{-}}^{V}}{\pi \alpha_{\mathrm{em}}} \\
& \times\left[\frac{F_{f_{1} N N}(t) \sqrt{1-t / 2 m_{V}^{2}}}{\left(Q^{2}+m_{V}^{2}-t\right)\left(t-m_{f_{1}}^{2}\right)}\right]^{2},
\end{aligned}
$$

where $F_{f_{1} N N}$ is the flavor singlet axial vector form factor of the nucleon. In Fig. 3 we present the calculated differential cross sections for vector meson $(\rho, \omega, \phi)$ photoproduction together with the experimental data [4, 14. The parameters are $\beta_{u}=\beta_{d}=2 \mathrm{GeV}^{-1}, \beta_{s}=1.5 \mathrm{GeV}^{-1}$ and $s_{0}=4$ $\mathrm{GeV}^{2}$.

One can see that our model reproduces the experimental data successfully. It should be mentioned that the $f_{1}$ contribution dominates over that of the Pomeron in the large $|t|$ region. This dominance is closely related to the slow decrease of the flavor singlet axial vector form factor of the nucleon with respect to $|t|$ as compared with that of the electromagnetic form factor, and to the different slopes of the trajectories.

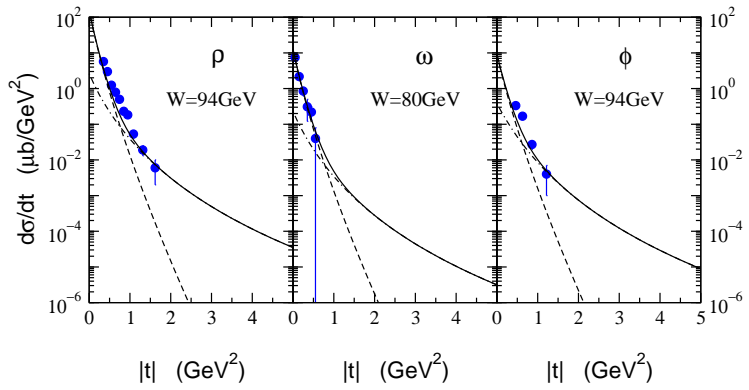

Figure 3. The differential cross sections for (a) $\rho$ meson at $W=94 \mathrm{GeV}$, (b) $\omega$ meson at $W=80$ $\mathrm{GeV}$ and (c) $\phi$ meson photoproduction at $W=94$ $\mathrm{GeV}$. The dashed and dot-dashed lines are the Pomeron and the $f_{1}$ exchange contributions, respectively, while the solid lines show the results by including the two exchanges. The experimental data are from Refs. [1, 14.

\section{3. $f_{1}$ contribution to vector meson photo- production in proton dissociation}

The recent report on vector meson photoproduction at large $t$ in proton dissociation by the ZEUS Collaboration [5] shows a surprising behaviour of the differential cross sections. The differential cross section data have very hard $t$ dependence. The observed data have $1 / t^{3}$ dependence instead of the $1 / t^{4}$ dependence which is expected from the pQCD approach. Within our model the dominant contribution to the proton dissociation at large $t$ comes from the $f_{1}$ exchange between the proton quarks and the quarkantiquark pair arising from the photon. It was mentioned that it would be a good approximation to consider the $f_{1}$-quark vertex as a pointlike vertex. Thus the main difference between the $f_{1}$ contributions to elastic [Eq. (3)] and dissociative production of vector mesons is the absence of the proton form factor in the formula for the differential cross section,

$\frac{d \sigma_{f_{1}}^{\text {diss }}}{d t d x}=\sum_{i} q_{i}(x, t) \frac{3 m_{V}^{3} g_{f_{1} N N}^{4} \Gamma_{e^{+} e^{-}}^{V}}{\pi \alpha_{\mathrm{em}}}$ 


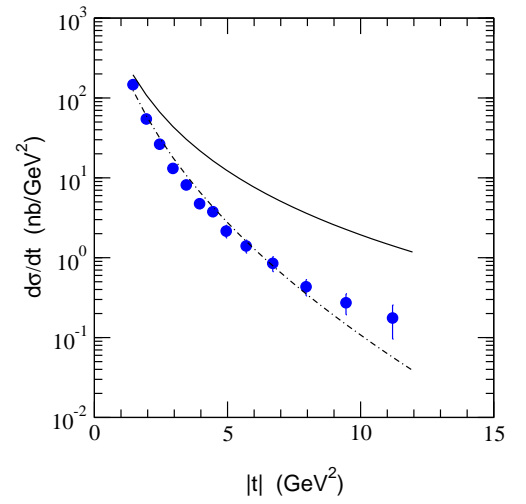

Figure 4. The differential cross section for $\rho$ meson photoproduction in proton dissociation process at $W=100 \mathrm{GeV}$. The solid and dashed lines are the $f_{1}$ exchange contribution with zero slope and with $\alpha_{f_{1}}^{\prime}=0.025 \mathrm{GeV}^{-2}$, respectively. The experimental data are from Ref. [5].

$$
\times\left[\frac{\sqrt{1-t / 2 m_{V}^{2}}}{\left(Q^{2}+m_{V}^{2}-t\right)\left(t-m_{f_{1}}^{2}\right)}\right]^{2},
$$

where $q_{i}(x, t)$ is the distribution of $i$-quark $(i=$ $u, d, s)$ in the proton, $x=-t /\left(M_{x}^{2}-t\right)$ and $M_{x}$ is the mass of the dissociative system. In Fig. we show the results of our calculation together with preliminary ZEUS data [5]. We have used the ZEUS cut $x>0.01$ and LO GRV98 15 partonic distributions. The agreement with the experimental data is rather good. The deviation at large $t$ may be due to the manifestation of nonzero (although small) slope of the $f_{1}$ trajectory (dashed line) or the deviation from the locality of the $f_{1}$-quark vertex.

\section{Summary}

We have calculated the $f_{1}$ anomalous trajectory contribution to vector meson electromagnetic production and have shown its important role in differential cross sections at large momentum transfers. The proton dissociation at large $t$ is one of the processes where the contribution from the $f_{1}$ exchange is very large. We have shown that the additional anomalous trajectory can explain preliminary ZEUS data reasonably.

N.I.K. would like to express his sincere gratitude to the Organizing Committee of the Diffraction 2000 Conference and especially to R. Fiore for the kind invitation and financial support. He is also grateful to many participants of the conference for fruitful discussions. This work was supported in part by the Brain Korea 21 project of Korean Ministry of Education.

\section{REFERENCES}

1. P. V. Landshoff, summary talk at Diffraction 2000, Centraro, Italy, hep-ph/0010315.

2. D. Kharzeev and E. Levin, Nucl. Phys. B578 (2000) 351; E. V. Shuryak, Phys. Lett. B486 (2000) 378.

3. M. M. Block, B. Margolis and A. R. White, talk at Blois 95, hep-ph/9510290.

4. ZEUS Collaboration, J. Breitweg et al., Eur. Phys. J. C14, 213 (2000).

5. ZEUS Collaboration, J. Breitweg et al., Paper 442, ICHEP2000, Osaka, Japan.

6. H1 Collaboration, C. Adloff et al., Phys. Lett. B483 (2000) 23.

7. N. I. Kochelev, D.-P. Min, Y. Oh, V. Vento and A. V. Vinnikov, Phys. Rev. D61 (2000) 094008.

8. L. Lukaszuk and B. Nicolescu, Nuovo Cimento Lett. 8 (1973) 55; A. Donnachie and P. V. Landshoff, Z. Phys. C2 (1979) 55.

9. Y. Oh, N. I. Kochelev, D.-P. Min, V. Vento and A. V. Vinnikov, Phys. Rev. D62 (2000) 017504.

10. A. B. Kaidalov and Yu. A. Simonov, Phys.Lett. B477 (2000) 163.

11. P. V. Landshoff and O. Nachtmann, Z. Phys. C35 (1987) 405; A. Donnachie and P. V. Landshoff, Phys. Lett. B 185 (1987) 403; Nucl. Phys. B311 (1988/89) 509.

12. J. H. Kühn and V. I. Zakharov, Phys. Lett. B252 (1990) 615.

13. A. Donnachie and P. V. Landshoff, Phys. Lett. B478 (2000) 146.

14. ZEUS Collaboration, M. Derrick et al., Z. Phys. C 73, 73 (1996).

15. M. Glück, E.Reya and A. Vogt, Eur. Phys. J. C5 (1998) 461. 